\title{
Automatic analysis of cross-sectional cerebral asymmetry on 3D in vivo MRI scans of human and chimpanzee
}

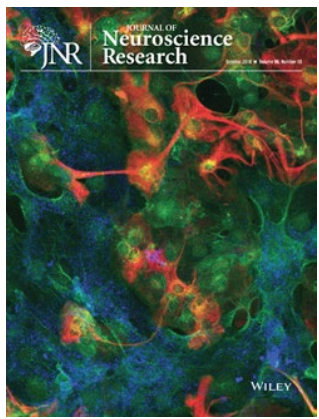

\section{\begin{tabular}{l|l|l} 
L. Xiang & (i) & T. J. Crow $^{2}$ \\
| N. Roberts
\end{tabular}}

\author{
${ }^{1}$ School of Clinical Sciences, University of \\ Edinburgh, Edinburgh, United Kingdom \\ ${ }^{2}$ University Department of \\ Psychiatry, Warneford Hospital, Oxford, \\ United Kingdom
}

\section{Correspondence}

L. Xiang, School of Clinical Sciences, University of Edinburgh, Edinburgh, EH16 4TJ, United Kingdom.

Email:xli9@ed.ac.uk

Funding information

University of Edinburgh

\begin{abstract}
One prominent feature of human brain asymmetry is the cerebral torque. To investigate whether this characteristic is shared with chimpanzees who are our closest extant relative, we developed an automatic method to compute cerebral hemisphere width and perimeter length on consecutive 2D sections through 3D MR images obtained in vivo for 91 human and 78 chimpanzee brains. In brief, contiguous inter hemispheric width and perimeter asymmetries were calculated on coronal sections, which in profile allow us to examine asymmetry in relation to speciation. The right frontal and left occipital asymmetry (greater posteriorly in females) distinguishes humans from chimpanzees. This result is consistent with a major saltational (discontinuity) event occurring at some point after the separation of humanity and the great apes in the last 6 million years.
\end{abstract}

KEYWORDS

brain, cerebral asymmetry, cerebral width, chimpanzee, speciation, torque

\section{1 | INTRODUCTION}

Cerebral dominance for certain human functions was first reported by Dax (1865) and Broca (1861) who described how the faculty of human speech is located in the left cerebral hemisphere. Since then the neuroanatomical asymmetries of Sylvian Fissure (Cunningham, 1892; Eberstaller, 1884, 1890; Rubens, Mahowald, \& Hutton, 1976), planum temporale (Geschwind \& Levitsky, 1968), and paracingulate sulcus (Paus et al., 1996) have been considered as possible structural substrates to account for functional laterality. The Yakovlevian torque (i.e. anticlockwise twist) is another prominent neuroanatomical asymmetry of the human brain. The pattern of the torque defines a 3D morphological twist, an archetypical view of which can be seen in Figure 2 of Toga and Thompson (2003). In particular, three principal features are observable on inspection of the external surface of the brain including (a) left-right tissue distribution asymmetry of wider frontal lobe on the right and wider occipital lobe on the left, (b) antero-posterior petalia referring to the protrusion of the right frontal and left occipital poles over the contralateral extremities, and (c) deviation (i.e. bending) of the inter-hemispheric fissure in the occipital region. Although the functional relevance of the torque is not yet understood, its correlation with asymmetry of the planum temporale (Barrick et al., 2005) and abnormalities in schizophrenia (Guerguerian \& Lewine, 1998; Maller et al., 2017), depression (Maller et al., 2014) and developmental stuttering (Foundas et al., 2003), suggest a possible relationship to cognitive function including perhaps the faculty of language (Best, 1988; Chance \& Crow, 2007; Crow, 2000, 2007, 2008, 2010; Esiri, Chance, de Barros, \& Crow, 2015; Galaburda, LeMay, Kemper, \& Geschwind, 1978).

Determining whether structural brain asymmetry is speciesspecific is essential in order to potentially identify its possible functional significance. The most direct approach to study the continuity/discontinuity of brain asymmetry is to compare humans with their closest extant relatives, great apes. However, inconsistent results have been reported in the evolution of cerebral asymmetry and which may be due to differences in materials, methods/measures, and cohorts. LeMay (1976) conducted the first investigation of whether the cerebral torque is present in nonhuman primates. From photographs of 28 great ape brains, leftward occipital width asymmetry and relatively greater posterior protrusion of the right 


\section{Significance}

Cerebral torque is the most prominent structural asymmetry of the human brain. The torque emerges early in the embryo and alternations of the torque have been linked to cognitive impairments in psychotic disorders, such as schizophrenia. The present study provides new evidence that the torque is specific to the human brain. Establishing the genetic origins of the torque may shed light on evolutionary theory, the origin of the psychiatric disorders and perhaps also the neural basis of language.

occipital pole were observed in apes, although in many cases the measurements of the left and right hemispheres were equal. In a MRI study, Hopkins and Marino (2000) measured the width of the cerebral hemispheres of 19 adult great apes, 15 adult Old and 8 New World monkeys. The width measurements were taken on the first axial 2D slice above the third ventricle at predefined locations in the frontal and occipital regions of the brain and defined as the distance between the Mid-Sagittal Plane (MSP) and the external surface. The authors reported typical right frontal and left occipital asymmetries in the great apes but not in monkeys. Balzeau, Gilissen, and Grimaud-Herve (2012) developed a method to compute the variation in the location of skull protrusions with respect to an external reference system on CT scans of endocasts of extant and fossil specimens (including 89 hominins and 110 African great apes). The authors reported significant antero-posterior and lateral components of the torque, especially posteriorly, in both humans $(p<0.01$, uncorrected) and great apes $(p<0.05$, uncorrected) and concluded that the torque is shared between humans and great apes. In contrast, in a study of a large collection of 190 hominoid endocasts, including specimens of 34 chimpanzees, 40 gorillas and 41 bonobos, Holloway and De La Costelareymondie (1982) did not observe the left occipital, right frontal petalia pattern in great apes at the group level and concluded that the cerebral torque developed early in hominid evolution. In a later MRI study of 9 post mortem adult chimpanzees and 29 living humans, Zilles et al. (1996) computed the magnitude of the relative displacement between the corresponding vertex pairs in left and mirrored right cerebral hemispheres. The authors reported the absence of torque in the chimpanzee brains. In our previous exploratory study of positional brain asymmetry (Xiang, Crow, Hopkins, Gong, \& Roberts, 2018), the variation in the location of the corresponding vertex pairs on the whole cerebral surface was automatically computed on MRI scans obtained in vivo for 223 live human and 70 chimpanzee brains. Three components of the inter-hemispheric displacement were measured (i.e. antero-posterior, dorso-ventral, and left-right) and a statistically significant pattern of torque, in particular, the petalia and bending, were observed in and only in the human brain. However, the width asymmetry favoring the right frontal and left occipital lobe was not observed in either humans or chimpanzees, possibly due to the simplification of the curved middle-sagittal surface by a plane, the location of which is essential to compute the relative deviation between the left and right cerebral hemispheres.

Analysis of left-right tissue distribution asymmetry between the two cerebral hemispheres has been commonly investigated in several studies by using the voxel based morphometry (VBM) technique (Barrick et al., 2005; Good et al., 2001; Hopkins et al., 2008; Luders, Gaser, Jancke, \& Schlaug, 2004; Watkins et al., 2001). In brief, after normalizing individual brains to the reference MNI template space, the inter-hemispheric asymmetry was computed as the gray matter density/volume difference between the left hemisphere and the mirror reflection of the right hemisphere across the MSP at corresponding voxel locations. The drawbacks of this approach are two-fold. Firstly, comparison of the voxels in the left and right cerebral hemispheres critically replies on the definition of the so-called MSP and, as mentioned above, approximation of this by the plane $x=0$, does not provide a reliable description of the curved interhemispheric fissure (Balzeau \& Gilissen, 2010; Xiang et al., 2018). Secondly, it is difficult to distinguish the tissue distribution asymmetry from the hemispheric shift (i.e. petalia), and which perhaps have different evolutionary origins (Chance \& Crow, 2007; Chance, Esiri, \& Crow, 2005). To overcome the problem, Chance et al. (2005) proposed a novel automatic approach in a postmortem MRI study of 23 patients with schizophrenia and 20 controls. In particular, section area was measured at 100 coronal slices evenly positioned through the left and right cerebral hemispheres so that the profiles obtained are independent of relative shifts of the hemispheres along the antero-posterior axis. However, the authors only reported the comparison at the slice with maximal cross-sectional area without analyzing the result for all slices.

In the present study, an automatic image analysis technique has been developed and applied to measure left and right tissue distribution asymmetry of the 3D external cerebral surface for in vivo MRI scans of 91 human and 78 chimpanzee brains. The algorithm is derived from the strategy used by Chance et al. (2005) in that contiguous inter-hemispheric width and perimeter asymmetries were calculated for 60 equidistant coronal sections through each cerebral hemisphere. On the basis of absence of torque characteristics in our previous studies (e.g. Xiang et al., 2018, Xiang, Crow, \& Roberts, 2019), we hypothesize that asymmetry in the tissue distribution will only be present in the human brain.

\section{2 | METHODS}

\section{1 | Subjects}

A sample of 91 healthy human subjects (mean age $34 \pm 12$ years, 39 females and 52 males) and 78 chimpanzees (mean age $24 \pm 11$ years, 50 females, and 28 males) were included in the study. Approval was obtained separately at each site from the local Research Ethics Committee and subjects provided fully informed written consent prior to taking part. The 3D MRI brain scans of human subjects were acquired at the Edinburgh Imaging facility 

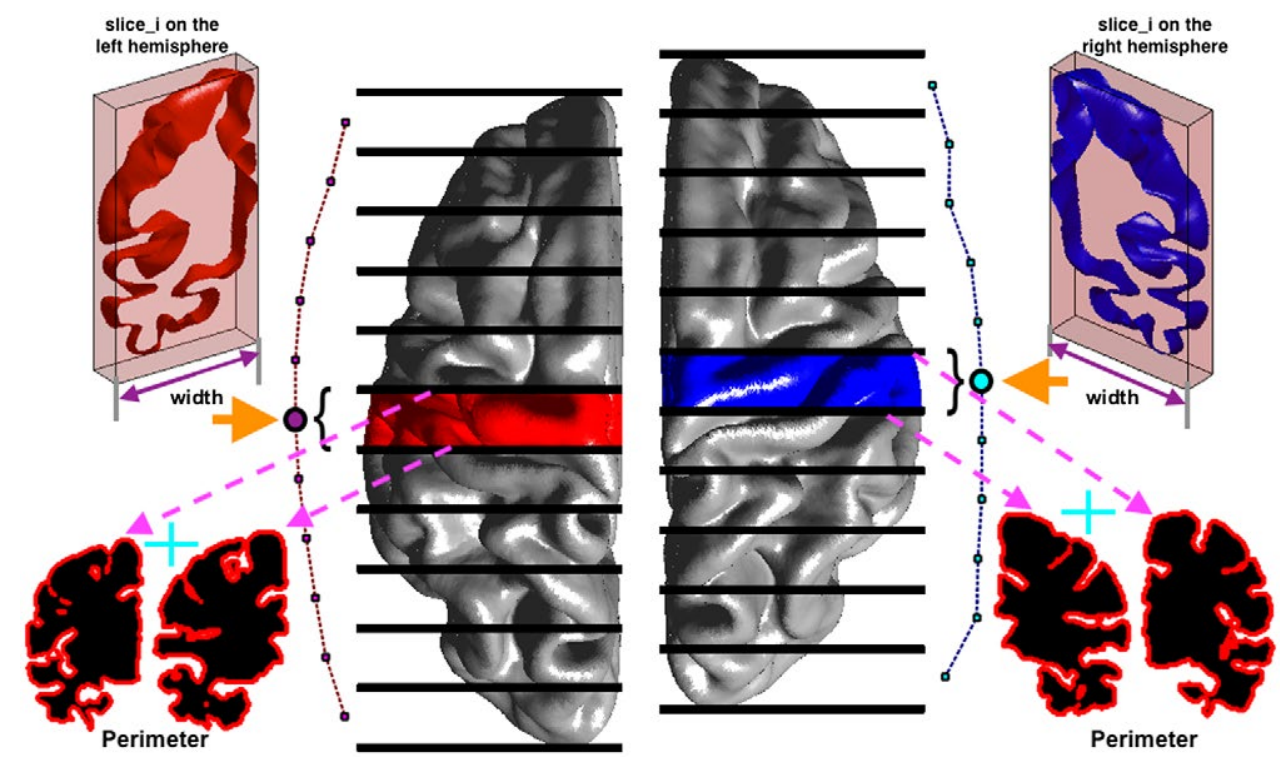

FIGURE 1 Computation of width and perimeter asymmetries. The width and perimeter of each cerebral hemisphere were measured at 60 contiguous slices orthogonal to the antero-posterior axis between the frontal and occipital poles. Asymmetries were computed as the difference between measurements obtained for the left and right cerebral hemispheres at each section

QMRI, University of Edinburgh, UK and the Oxford Centre for Magnetic Resonance, University of Oxford, UK. In the Oxford cohort, the Annett Handedness Inventory score was recorded for 47 subjects in which 2 are left-handed. In the Edinburgh cohort, the handedness score was recorded for 31 subjects, in which 4 are left-handed and 2 have ambiguous handedness. Therefore, the proportion of subjects who are right handed lies between $80.7 \%$ and $95.7 \%$. For the human subjects studied in Edinburgh, the images were acquired using a 3D magnetization-prepared rapid acquisition gradient echo (MPRAGE) sequence on a $3 \mathrm{~T}$ Verio MRI system (Siemens Medical Systems, Erlangen, Germany). Acquisition parameters were $\mathrm{TR}=2,300 \mathrm{~ms}, \mathrm{TE}=2.98 \mathrm{~ms}, \mathrm{TI}=900 \mathrm{~ms}$, Flip angle $=9^{\circ}, \mathrm{FOV}=256 \mathrm{~mm} \times 256 \mathrm{~mm}$ with an isotropic voxel resolution of $1 \mathrm{~mm}$. For the human subjects studied in Oxford, the images were acquired using a 3D fast low-angle shot (FLASH) sequence on a Sonata 1.5 T MR system (Siemens Medical, Erlangen, Germany). Acquisition parameters were $T R=5,400 \mathrm{~ms}$, $\mathrm{TE}=76 \mathrm{~ms}$, Flip angle $=90^{\circ}, 256 \times 160$ slice matrix comprising 208 contiguous slices with an isotropic voxel resolution of $1 \mathrm{~mm}$.

MRI scanning of the 78 chimpanzees (50 females, 28 males) was performed at Yerkes National Primate Research Centre in Atlanta, Georgia, Unite States. Chimpanzees were immobilized by ketamine injection $(10 \mathrm{mg} / \mathrm{kg})$ and subsequently anesthetized with propofol (40-60 $\mathrm{mg} \mathrm{kg}^{-1} \mathrm{hr}^{-1}$ ) before transportation to the MRI facility where they remained anesthetized (total time $\sim 2 \mathrm{hr}$ ) for the MR imaging and return to the home compound. They were scanned supine with a human head coil. T1-weighted MPRAGE MR images were obtained using a Siemens 3 T Trio MR system. Acquisition parameters were $\mathrm{TR}=2,300 \mathrm{~ms}, \mathrm{TE}=4.4 \mathrm{~ms}, \mathrm{TI}=1,100 \mathrm{~ms}$, flip angle $=8$, FOV $=200 \mathrm{~mm} \times 200 \mathrm{~mm}$. The data matrix size was $320 \times 320$ with an isotropic voxel resolution of $0.6 \mathrm{~mm}$.

\section{2 | Image analysis}

All MRI scans were pre-processed in FSL (http://sl.fmrib.ox.ac.uk/fsl/ fslwiki/) including skull strip, bias field correction, and brain normalization to the MNI152 template using 7 degrees of freedom (DoF) transformations (i.e. 3 translations, 3 rotations, and 1 uniform scaling). Details of the processes are described elsewhere (Hopkins, Li, Crow, \& Roberts, 2016). The 7 DoF transformation is particularly important to normalize the chimpanzee brain to the size of the human brain to enable to subsequent processing in the standard FreeSurfer processing stream that is designed for the human brain (https://surfer.nmr.mgh.harvard.edu/). The uniform scaling is key to preserve the morphological shape of the cerebral surface. In FreeSurfer, the bias field correction was repeated to assist the classification of the voxel data into different tissue types and to locate the boundaries between different brain tissues. The white matter is labelled, split into two cerebral hemispheres with the cerebellum and brain stem removed. A triangular mesh is fitted to cover the outer voxels of the connected white matter component for each cerebral hemisphere and deformed with respect to the intensity gradient between the white and gray matter to produce a final mesh surface with sub-voxel resolution. The resulting surface representing the boundary between the cerebral cortex and underlying white matter is expanded along the direction of the image signal intensity gradient between gray matter and CSF until it coincides with the pial surface and topologically corrected (Dale, Fischl, \& Sereno, 1999). The quality of reconstructed cerebral surface is essential to the subsequent measurement and was visually inspected. According to our previous work (Xiang et al., 2018), the extraction of the external surface of the cerebral hemispheres using FreeSurfer software was consistent in subjects scanned on different MR systems and therefore the MR system had no effect on the pattern of brain asymmetry identified in different cohorts. 

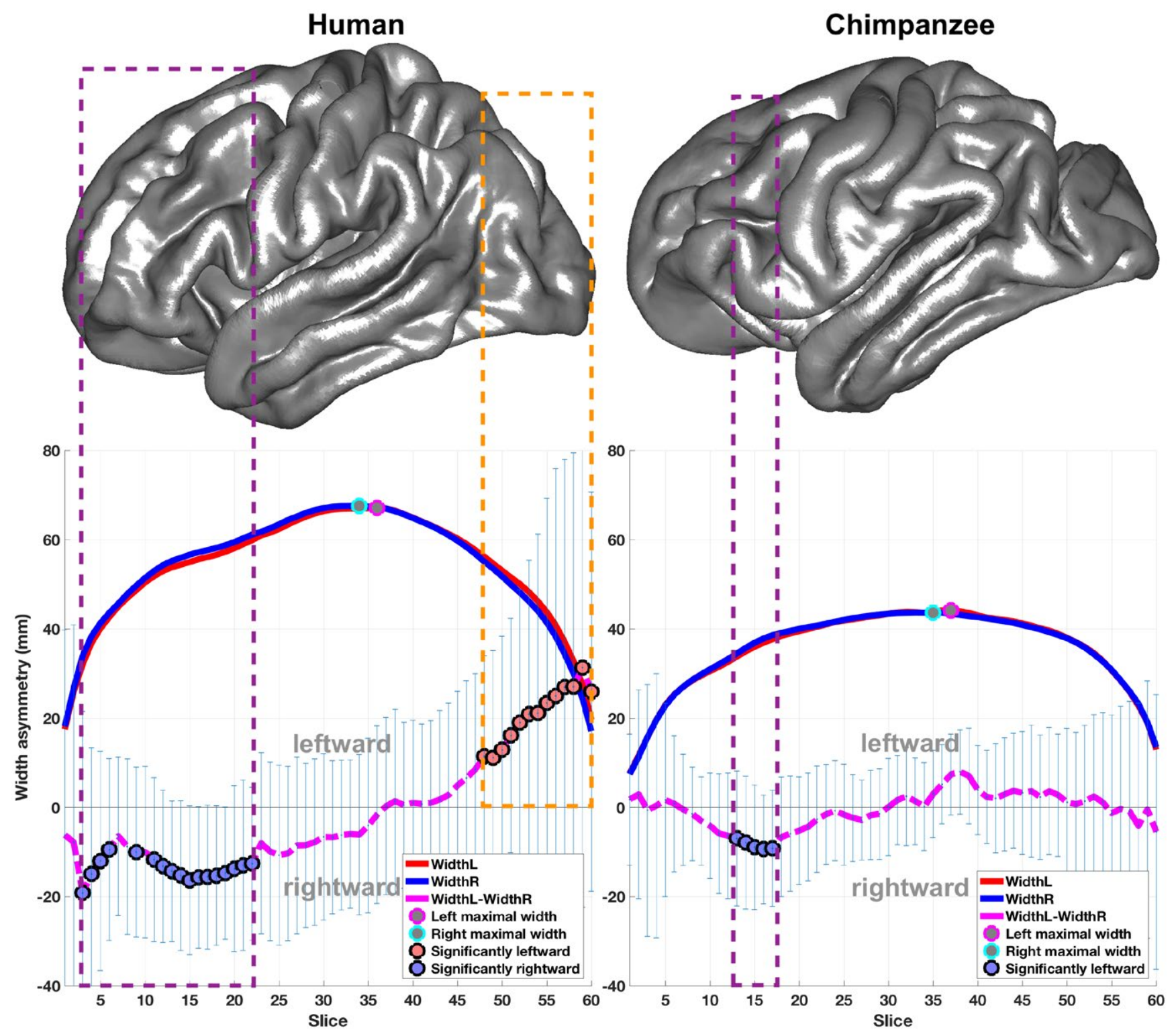

FIGURE 2 Hemispheric width asymmetry profile of the human (left) and chimpanzee (right) brain. The $x$-coordinate corresponds to brain section number along the antero-posterior axis. The $y$-coordinate corresponds to the width difference between the two cerebral hemispheres (in magenta) with error bar computed as the standard deviation from the average asymmetry at the associated slice as well as the lateral-medial widths for the left (in red) and right (in blue) hemispheres, respectively. Positive values indicate leftward asymmetry and negative values indicate rightward asymmetry. The purple and orange circles on the average width difference profile highlight the slices where the asymmetry is significant $(p<0.01 / 60)$ and the normalized inter-hemispheric difference is greater than $2 \%$. The slice with largest width is denoted by a magenta circle for the left cerebral hemisphere and a cyan circle for the right. A lateral view of the average surface of the left cerebral hemisphere of each species is displayed above the width plots. The segments of the profile which exhibit significant width asymmetries are marked by dashed lines. The width values of human and chimpanzee brains reflect the brain size in native space. In order to present the width and width asymmetry in the same plot, the width difference value is multiplied by 10

When the brain is asymmetric, the low-dimensional linear registration in FSL is often insufficient in aligning the center of the brain to the center of the MNI reference space (the plane $x=0$ ). In order to obtain accurate brain measurements, the orientation of the reconstructed cerebral surface from FreeSurfer was modified as follows: (a) the center of the brain was estimated as the 3D MSP which best fits the vertices on the medial surface of the brain lying within $5 \mathrm{~mm}$ of $x=0$, so as to limit the inclusion of regions where there are substantial deviation (e.g., occipital falx). (b) The MSP is then aligned with $x=0 \mathrm{~mm}$ so that the MSP of each brain was closely aligned with the MSP of the MNI152 template. More details can be found in Xiang et al. (2018).

The left-right tissue distribution asymmetry was measured on a cross-sectional basis using a program written in-house in Matlab (Version R2011b) software by LX. As demonstrated in Figure 1, each cerebral hemisphere was independently and evenly cut into identical 
number of slices (i.e., 60) perpendicular to the antero-posterior axis. For each slice, a minimal rectangular box covering the slice surface was generated with three edges parallel to the $x, y$, and $z$ axes. The width of cerebral hemisphere at each slice corresponds to the width of the associated bounding box (see Figure 1 (top left and top right)). The perimeter of the external cerebral hemisphere was measured at two ends of each slice as the average of the length of the brain outlines (see Figure 1 (bottom left and bottom right)). The profile of both width and perimeter measures for the slices represents the variation of the hemisphere external morphology along the antero-posterior direction. The difference of the measures between the left and right hemispheres reflected the bilateral variation of the cerebral surface.

The FreeSurfer pipeline also provides measures of the global and lobar brain surface areas for each species. In particular, by employing the so-called "Desikan-Killiany" atlas (Desikan et al., 2006), the surface of each cerebral hemisphere can be independently subdivided into a number of local regions (i.e., 34 regions of interest), and some regions can be merged together to form individual lobes, for which the total surface area can also be computed. All the measurements were corrected to reflect true size in real world using the uniform scaling factor previously computed in FSL.

\section{3 | Statistical analysis}

At each location along the antero-posterior axis, the width and perimeter of the left and right hemispheres were compared using paired two-sample $t$ tests (two-tailed). The locations (i.e., slices) were considered to be significantly asymmetric only if the normalized interhemispheric difference was greater than $2 \%$ and the result passed the significance level alpha of 0.01 and survived the Bonferroni correction for multiple comparisons. The sex difference of the width and perimeter asymmetries was examined using two-sample $t$ tests (two-tailed) with alpha set to 0.05 without multiple corrections. A lower threshold was used for the analysis of sex-differences on account of the relatively small size of the groups.

The global and lobar brain surface areas were compared between hemispheres using paired two-sample $t$ tests (two-tailed) and Bonferroni corrected for multiple comparisons. The effect of sex and age on the asymmetry was tested using multivariate analysis of variance (MANCOVA) with inter-hemispheric difference as dependent variable, sex as between group factor and age as covariate.

\section{3 | RESULTS}

\subsection{Brain global and lobar surface area}

From Table 1, the right hemisphere of the human brain showed significantly greater surface area than the left both globally $(t=-4.10, p<0.001)$ and in the frontal $(t=-4.27, p<0.001)$, parietal $(t=-9.26, p<0.001)$, occipital $(t=-2.48, p=0.015)$, and insula $(t=-5.22, p<0.001$ ) lobes, but not in the temporal lobe $(t=10.19, p<0.001)$ where a leftward asymmetry was found. The results survived Bonferroni corrected for multiple comparisons.

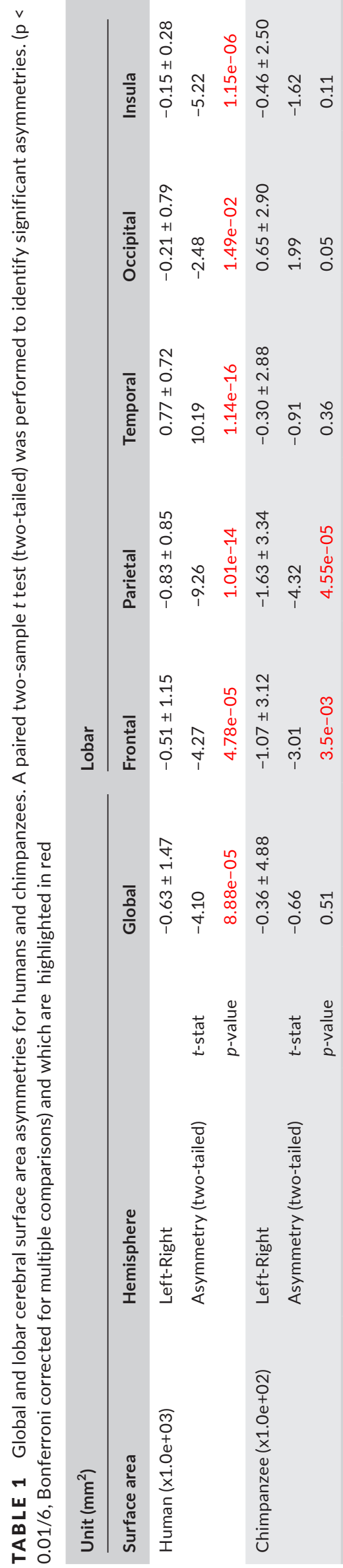


In contrast, there was no significant global surface area asymmetry in the chimpanzee brain. However, rightward asymmetry was found in the frontal $(t=-3.01, p<0.001)$ and parietal $(t=-4.32$, $p<0.001)$ lobes. According to MANCOVA tests, neither sex $(F(6,83)=0.56, p=0.763)$ or age $(F(6,83)=1.20, p=0.314)$ showed significant effect on the asymmetries in humans and similar results $(F(6,63)=1.46, p=0.206$ for $\operatorname{sex} ; F(6,63)=1.59, p=0.164$ for age $)$ were found in chimpanzees.

\subsection{Cross-sectional width and perimeter asymmetries}

The slice-wise inter-hemispheric width difference together with the width measures of each hemisphere over the antero-posterior expansion are displayed in Figure 2. From the left panel, human width asymmetries, referring to the magenta dot plot, showed a significant right frontal and left occipital pattern of torque. The anterior part of the brain from the frontal pole to the precentral gyrus demonstrated a rightward asymmetry and the posterior part of the brain from the parietal lobe to the occipital pole showed a leftward asymmetry, denoted by the orange circles above the width differential profile. The direction of width asymmetry changed from right to left at about the 42nd slice which is in the region of Heschl's gyrus. In addition, the slice with maximal width is positioned significantly more posterior on the left ( 36th slice) compared to the right ( 34th slice) according to paired $t$ tests $t(90)=3.20, p=0.002$, one-tailed. According to paired $t$ tests, the maximal width of the right hemisphere $(68.2 \pm 3.2 \mathrm{~mm})$ is significantly larger than that of the left $(67.8 \pm 2.8 \mathrm{~mm}),(t(90)=-1.82$, $p=0.036$, one-tailed). Furthermore, according to Pearson's correlation test, the difference between the maximal width of the left and right hemispheres is positively correlated with global inter-hemispheric surface area asymmetry by a coefficient of 0.21 $(p=0.041)$

In the case of the chimpanzee brain the right frontal and left occipital pattern of width asymmetry is not present in the chimpanzee
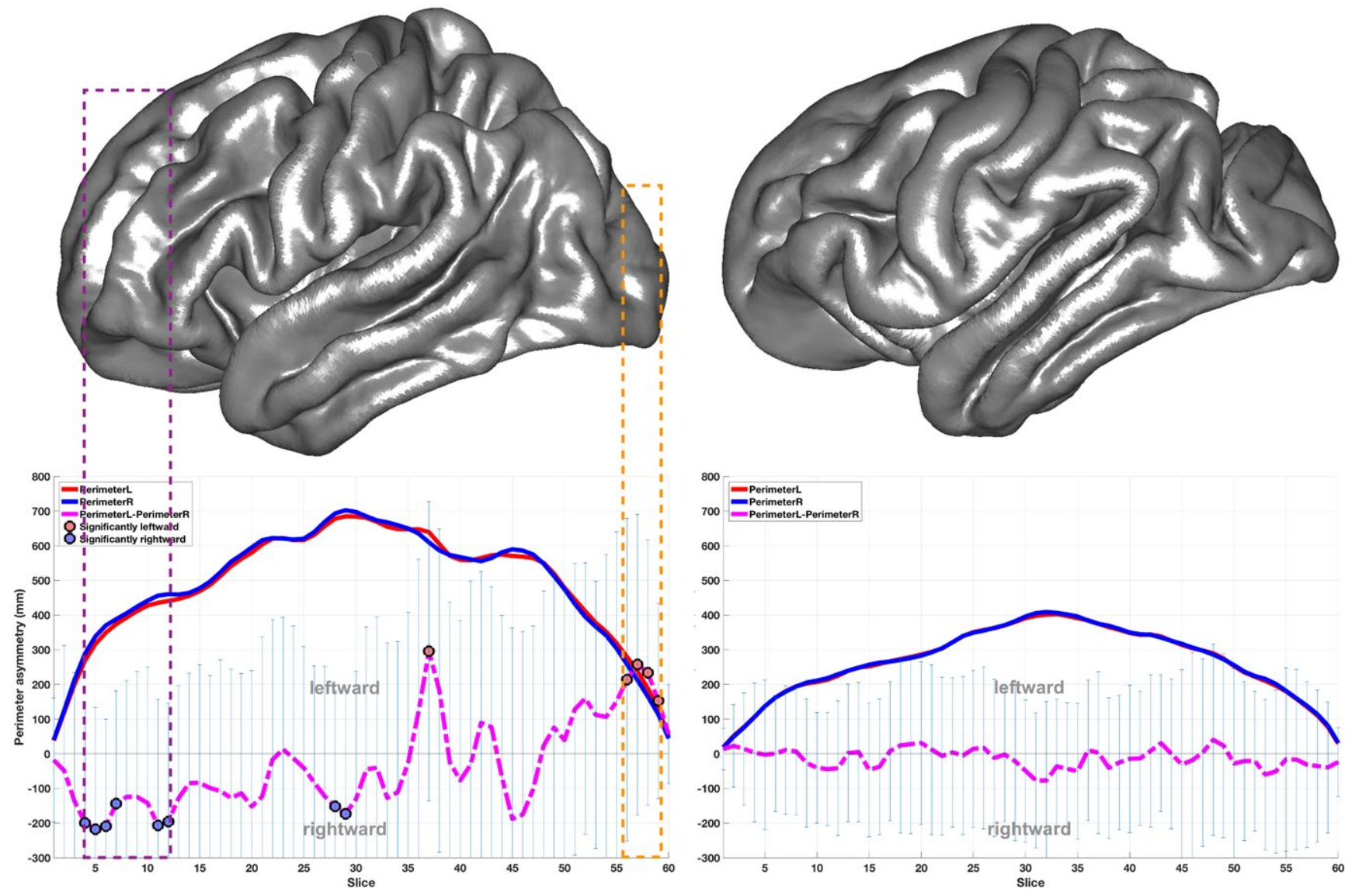

FIGURE 3 Hemispheric perimeter asymmetry profile of the human (left) and chimpanzee (right) brain. The $x$-coordinate corresponds to brain section number along the antero-posterior axis. The $y$-coordinate corresponds to the perimeter difference between the two cerebral hemispheres with error bar computed as the standard deviation from the average asymmetry at the associated section as well as the perimeter profiles for the left (in red) and right (in blue) hemispheres, respectively. Positive asymmetry indicates a leftward perimeter asymmetry while negative for rightward. The purple and orange circles on the average width difference profile highlight the slices where the asymmetry is significant $(p<0.01 / 60)$ and the normalized interhemispherical difference is greater than $2 \%$. A lateral view of the average surface of the left cerebral hemisphere of each species is displayed above the width plots. The segments of the profile which exhibit significant width asymmetries are marked by dashed lines. The values of human and chimpanzee brains reflect the brain size in native space. In order to present the perimeter and perimeter asymmetry in the same plot, the perimeter difference value was multiplied by 10 

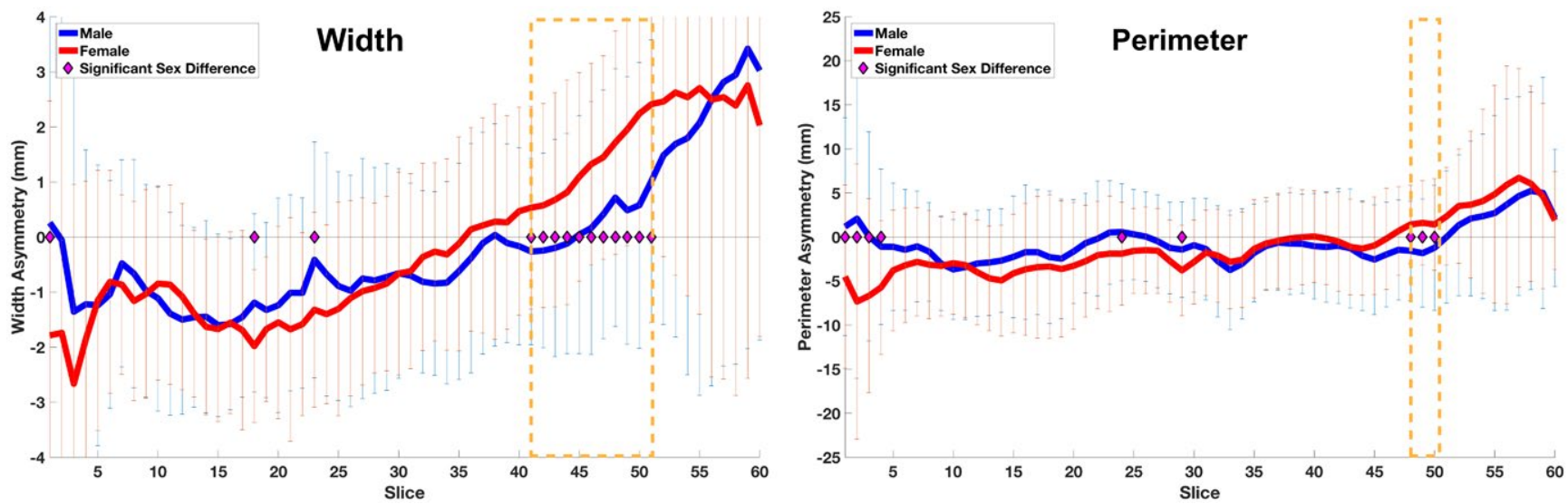

FIGURE 4 The sex difference of left-right width (left panel) and perimeter length (right panel) asymmetries in humans. The $x$-coordinate denotes the brain number along the antero-posterior axis. The $y$-coordinate corresponds to the inter-hemispheric difference with error bar computed from standard deviation from the average asymmetry at the associated slice location with female in red and male in blue. Positive values indicate leftward asymmetry and negative values rightward asymmetry. The magenta diamond refers to sections where the sex difference is significant $(p<0.05)$. The chimpanzees show no sex difference in either width or perimeter asymmetry

brain (see Figure 2, right panel). In particular, the width difference between hemispheres is completely absent in the occipital lobe although interestingly, a short segment in the region of Broca's area showed asymmetries resembling in part the rightward frontal width asymmetry of the human brain. Similar to that of the human brain, the slice with maximal width significantly shifted posteriorly in the left (37th slice) compared the right (35th slice) according to paired $t$ tests $t(77)=5.30, p<0.001$, one-tailed. The maximal width is $44.5 \pm 2.0 \mathrm{~mm}$ in the left cerebral hemisphere and $44.1 \pm 2.0 \mathrm{~mm}$ on the right. Significant leftward rather than rightward asymmetry was found between the maximal width measures of the two hemispheres $(t(77)=4.00, p<0.001$, one-tailed $)$.

The inter-hemispherical perimeter asymmetry along the anteroposterior axis and the perimeter profile of each hemisphere for both species are depicted in Figure 3. Similar to the result of the width measurements, a greater right frontal and left occipital torque pattern was observed in the human brain but not the chimpanzee.

\subsection{Sex difference}

After the sexes were separated, human females showed a significantly increased leftward width asymmetry posteriorly (Figure 4, left panel) relative to males $(p<0.05)$. Similarly, sexual dimorphism was also found in the perimeter asymmetry measure $(p<0.05)$, which overlaps with the region found in the width asymmetry measure (Figure 4, right panel). For chimpanzees no significant sex difference in width or perimeter asymmetry was found.

\section{4 | DISCUSSION}

The results of the present study confirm that opposing asymmetry in the frontal and occipital regions, typically referred to as the cerebral torque pattern, is present only in the human brain. In addition, the location of the slice with the maximal width was found to be more posterior in the left hemisphere compared to the right cerebral hemisphere and which is compatible with the greater posterior sheer of the temporal and occipital regions of the left cerebral hemisphere reported in our previous work (Xiang et al., 2018). On the contrary, profiles of both the cross-sectional width and perimeter appeared identical in both cerebral hemispheres of the chimpanzee brain. These finding do not support the reports of the presence of the torque in great ape brains (Hopkins \& Marino, 2000; LeMay, Billig, \& Geschwind, 1982) but is consistent with studies that concluded that the brain asymmetry is a new feature in the hominin lineage (Holloway \& De La Costelareymondie, 1982; Xiang et al., 2018, 2019; Zilles et al., 1996).

Some of the differences between the results from previous studies may relate to the fact that different methods have been used and where the measurement was performed on 2D axial sections (Guerguerian \& Lewine, 1998; Hopkins \& Marino, 2000), the result is less likely to accurately represent the morphology of 3D features. Furthermore, in some studies the measurement of lateral tissue distribution asymmetry did not control for the hemispheric shift in the antero-posterior direction (Barrick et al., 2005; Good et al., 2001; Hopkins et al., 2008; Luders et al., 2004; Watkins et al., 2001). In other cases where the asymmetries were computed with respect to the inter-hemispheric fissure, the approximation of the curved surface by a $2 \mathrm{D}$ line or $3 \mathrm{D}$ plane is likely to introduce inaccuracy in the measurement (Hopkins et al., 2008; Xiang et al., 2018). In addition, study of different types of specimens may lead to different results. For example, even though they serves as a good proxy for brain shape, there is incomplete correspondence between endocranial casts taken from skulls and the brain (Balzeau \& Gilissen, 2010; Holloway \& De La Costelareymondie, 1982; LeMay, 1976) and shrinkage and distortion of postmortem brains (Hopkins \& Marino, 
2000; Hopkins et al., 2008) may alter brain morphology. The advantage of the present study is three-fold. Firstly, the relative anteroposterior shift of the right and left cerebral hemispheres has been controlled for and the measurement of width and perimeter at each cross section is independent of the location of the MSP. Secondly, it is advantageous to perform the same analysis on MR images in vivo for both the chimpanzee and human brains obtained using an almost identical protocol. Thirdly, the asymmetry of the external surface of the brain was computed fully automatically without human intervention and therefore the result is reproducible for any individual subject. Overall, this comparison study of relatively large cohorts of 91 human and 78 chimpanzee brains challenges the view that the former species has undergone similar asymmetric re-organizations as the later and casts doubt on the suggestion that tissue distribution asymmetry is an attribute of the common ancestor of humans and great apes.

Language and average right handedness have been suggested to be unique to the human species (Chance \& Crow, 2007; Warren, 1980), and so, according to our recent findings (Xiang et al., 2018, and the present study) is the cerebral torque. Gratiolet and Leuret (1839) reported there to be a greater gyrification of the left frontal and right occipital regions relative to the contralateral hemisphere and Best (1988) later proposed the existence of three growth gradients that emerge early in the embryo. The growth gradients along the dorso-ventral and antero-posterior axes have been found to be specific in the human brain in our previous work (Xiang et al., 2019). The present study additionally suggests that asymmetry in the leftright direction is also of late origin in the past 6 million years.

Sex difference of brain asymmetry along the antero-posterior axis has been reported previously. For example, Guerguerian and Lewine (1998) reported that for healthy control subjects males showed reduced torque compared to females whereas for patients with schizophrenia males showed increased torque compared to females. Highley et al. (1998) reported that asymmetry of the length from the frontal pole to the central sulcus measured dorsally over the external surface of the brain on both hemispheres, showed a gender by diagnosis interaction. Female controls had a left-greaterthan-right asymmetry, and male controls had a right-greater-thanleft asymmetry. This pattern was reversed in schizophrenia. In the present study, a sex difference was observed in the posterior parietal and occipital regions such that greater leftward asymmetry was found in females than males, in agreement with the observation of (Guerguerian \& Lewine, 1998).

One limitation of the present study is the lack of full knowledge of handedness in the healthy participants, although no significant association was identified between brain asymmetries and handedness in a meta-analysis of a large database of 17,141 healthy individuals (Kong et al., 2018). In addition, the finding in the present study was based on the captive Yerkes chimpanzee group, which may not fully reflect the natural populations of chimpanzees and other great apes. With regard to the asymmetries found in the frontal and parietal lobes in the chimpanzee brains, the effect of the human template bias derived the FreeSurfer surface parcellation scheme needs further evaluation (Kong et al., 2018).

In conclusion, the results of the present study show that asymmetry favoring the right frontal and left occipital regions of the brain is a characteristic that separates humans from chimpanzees and may thus be considered a species-defining characteristic, or autapomorphy. The finding provides new evidence to support a saltational jump as compared to the classical Darwinian concept of gradual transition in speciation and therefore the study has implications for evolutionary theory.

\section{ACKNOWLEDGMENTS}

We thank Prof. William D. Hopkins for access to MRI scans of chimpanzees at the Yerkes National Primate Centre and Prof. Stephen Lawrie for access to MRI scans of humans at the University of Edinburgh and staff at the University of Oxford for help in scanning procedures and for access to the series of MRI scans from normal human subjects. This research was supported by the T.J. Crow Psychosis Research Trust.

\section{CONFLICT OF INTEREST}

The authors declared that they have no conflict of interest.

\section{AUTHOR CONTRIBUTIONS}

Analysis and interpretation of data: X.L, N.R and T.J.C.; Statistical analysis: X.L.; Writing, Review \& Editing: X.L., N.R. and T.J.C. All authors reviewed and approved the paper.

\section{ORCID}

L. Xiang (iD https://orcid.org/0000-0003-3846-1374

\section{REFERENCES}

Balzeau, A., \& Gilissen, E. (2010). Endocranial shape asymmetries in Pan paniscus, Pan troglodytes and Gorilla gorilla assessed via skull based landmark analysis. Journal of Human Evolution, 59(1), 54-69. https:// doi.org/10.1016/j.jhevol.2010.03.013

Balzeau, A., Gilissen, E., \& Grimaud-Herve, D. (2012). Shared pattern of endocranial shape asymmetries among great apes, anatomically modern humans, and fossil hominins. PLoS ONE, 7(1), e29581. https://doi.org/10.1371/journal.pone.0029581

Barrick, T. R., Mackay, C. E., Prima, S., Maes, F., Vandermeulen, D., Crow, T. J., \& Roberts, N. (2005). Automatic analysis of cerebral asymmetry: An exploratory study of the relationship between brain torque and planum temporale asymmetry. Neurolmage, 24(3), 678-691. https:// doi.org/10.1016/j.neuroimage.2004.09.003

Best, C. T. (1988). The emergence of cerebral asymmetries in early human development: A literature review and a neuroembryological model. In D. L. Molfese \& S. J. Segalowitz (Eds.), Brain lateralization in children: Developmental implications (pp. 5-34). New York, NY: Guilford.

Broca, P. (1861). Remarques sur la siege de la faculte du langue (Remarks on the Seat of the Faculty of Articulated Language). Bulletin De La 
Societee Anatomique De Paris (2nd series), 6, 330-357. https://doi. org/10.1093/acprof:oso/9780195177640.003.0018

Chance, S. A., \& Crow, T. J. (2007). Distinctively human: Cerebral lateralisation and language in Homo sapiens. Journal of Anthropological Sciences, 85, 83-100.

Chance, S. A., Esiri, M. M., \& Crow, T. J. (2005). Macroscopic brain asymmetry is changed along the antero-posterior axis in schizophrenia. Schizophrenia Research, 74(2-3), 163-170. https://doi.org/10.1016/j. schres.2004.09.001

Crow, T. J. (2000). Schizophrenia as the price that Homo sapiens pays for language: A resolution of the central paradox in the origin of the species. Brain Research Reviews, 31(2-3), 118-129. https://doi. org/10.1016/s0165-0173(99)00029-6

Crow, T. J. (2007). Nuclear schizophrenic symptoms as the key to the evolution of the human brain. In J. H. Kaas (Ed.), Evolution of Nervous Systems (1st ed., Vol. 4, pp. 549-567). London, UK: Elsevier. (Reprinted from: NOT IN FILE).

Crow, T. J. (2008). The torque defines the four quadrants of the human language circuit and the nuclear symptoms of schizophrenia identify their component functions. In B. Stemmer \& H. A. Whittaker (Eds.), Handbook of the neurocience of language (Vol. 1, pp. 299-307). London, UK: Whittaker Academic Press. (Reprinted from: NOT IN FILE).

Crow, T. J. (2010). The nuclear symptoms of schizophrenia reveal the four quadrant structure of language and its deictic frame. Journal of Neurolinguistics, 23(1), 1-9. https://doi.org/10.1016/j. jneuroling.2009.08.005

Cunningham, D. J. (1892). Contribution to the surface anatomy of the cerebral hemispheres. Cunningham Mem. (R. Ir., Acad.), 7(372).

Dale, A. M., Fischl, B., \& Sereno, M. I. (1999). Cortical surface-based analysis I: Segmentation and surface reconstruction. Neurolmage, 9(2), 179-194. https://doi.org/10.1006/nimg.1998.0395

Dax, M. (1865). Lesions de la moitie gauche de l'encephale coincident avec l'oubli des signes de la pensee (Read at congres meridional at Montpelier in 1836). GazHebdomMedChirurg, 11, 259-260. English translation in Joynt J, Benton AL: The memoir of Marc Dax on aphasia. Neurology 1964;14:851-854.

Desikan, R. S., Segonne, F., Fischl, B., Quinn, B. T., Dickerson, B. C., Blacker, D., \& Killiany, R. J. (2006). An automated labeling system for subdividing the human cerebral cortex on MRI scans into gyral based regions of interest. Neurolmage, 31(3), 968-980. https://doi. org/10.1016/j.neuroimage.2006.01.021

Eberstaller, O. (1884). Z ü $r$ Oberflachen Anatomie der Grosshirn (Surface anatomy of the cerebrum). Hemisphaeren. Wien Med BI, 7(479), 642-644.

Eberstaller, O. (1890). Das Stirnhirn. Ein Beitrag zur Anatomie der Oberfläche des Grosshirns (The forebrain. A contribution to the anatomy of the surface of the cerebrum). Wien und Leipzig: Urban and Schwarzenberg.

Esiri, M. M., Chance, S. A., de Barros, J., \& Crow, T. J. (2015). Psychiatric diseases. In S. Love, A. Perry, J. Ironside \& H. Budka (Eds.), Greenfield's neuropathology (Vol. 9, pp. 975-1007). London, UK: Taylor and Francis.

Foundas, A. L., Corey, D. M., Angeles, V., Bollich, A. M., Crabtree-Hartman, E., \& Heilman, K. M. (2003). Atypical cerebral laterality in adults with persistent developmental stuttering. Neurology, 61(10), 1378-1385. https://doi.org/10.1212/01.WNL.0000094320.44334.86

Galaburda, A. M., LeMay, M., Kemper, T. L., \& Geschwind, N. (1978). Right-left asymmetries in brain. Science, 199(4331), 852-856. https:// doi.org/10.1126/science.341314

Geschwind, N., \& Levitsky, W. (1968). Human brain: Left-right asymmetries in temporal speech region. Science, 161(3837), 186-187. https:// doi.org/10.1126/science.161.3837.186

Good, C. D., Johnsrude, I., Ashburner, J., Henson, R. N., Friston, K. J., \& Frackowiak, R. S. (2001). Cerebral asymmetry and the effects of sex and handedness on brain structure: A voxel-based morphometric analysis of 465 normal adult human brains. Neurolmage, 14(3), 685700. https://doi.org/10.1006/nimg.2001.0857

Gratiolet, P., \& Leuret, F. (1839). Anatomie compare du systeme nerveux, considere dans ses rapports avec l'intelligence (Anatomy comparison of the nervous system, considering its role in intelligence). Paris, France: J.B. Bailliere et Fils.

Guerguerian, R., \& Lewine, R. R. J. (1998). Brain torque and sex differences in schizophrenia. Schizophrenia Research, 30(2), 175-181. https://doi.org/10.1016/S0920-9964(97)00144-8

Highley, J. R., Esiri, M. M., McDonald, B., Cortina-Borja, M., Cooper, S. J., Herron, B. M., \& Crow, T. J. (1998). Anomalies of cerebral asymmetry in schizophrenia interact with gender and age of onset: a post-mortem study. Schizophrenia Research, 34(1-2), 13-25.

Holloway, R. L., \& De La Costelareymondie, M. C. (1982). Brain endocast asymmetry in pongids and hominids: Some preliminary findings on the paleontology of cerebral dominance. American Journal of Physical Anthropology, 58(1), 101-110. https://doi.org/10.1002/ ajpa.1330580111

Hopkins, W. D., Li, X., Crow, T., \& Roberts, N. (2016). Vertex- and atlasbased comparisons in measures of cortical thickness, gyrification and white matter volume between humans and chimpanzees. Brain Structure and Function, 222(1), 229-245. https://doi.org/10.1007/ s00429-016-1213-1

Hopkins, W. D., \& Marino, L. (2000). Asymmetries in cerebral width in nonhuman primate brains as revealed by magnetic resonance imaging (MRI). Neuropsychologia, 38(4), 493-499. https://doi.org/10.1016/ S0028-3932(99)00090-1

Hopkins, W. D., Taglialatela, J. P., Meguerditchian, A., Nir, T., Schenker, N. M., \& Sherwood, C. C. (2008). Gray matter asymmetries in chimpanzees as revealed by voxel-based morphometry. Neurolmage, 42(2), 491-497. https://doi.org/10.1016/j. neuroimage.2008.05.014

Kong, X. Z., Mathias, S. R., Guadalupe, T., Glahn, D. C., Franke, B., Crivello, F., \& Francks, C. (2018). Mapping cortical brain asymmetry in 17,141 healthy individuals worldwide via the ENIGMA consortium. Proceedings of the National Academy of Sciences, 115(22), E5154-e5163. https://doi.org/10.1073/pnas.1718418115

LeMay, M. (1976). Morphological cerebral asymmetries of modern man, fossil man, and non-human primate. Annals of the New York Academy of Sciences, 280, 349-369. https://doi.org/10.1111/j.1749-6632.1976. tb25499.x

LeMay, M., Billig, M. S., \& Geschwind, N. (1982). Asymmetries of the brains and skulls of nonhuman primates. In D. F. E. Armstrong (Ed.), Primate brain evolution. Methods and concepts (pp. 263-277). New York, NY: Plenum Press.

Luders, E., Gaser, C., Jancke, L., \& Schlaug, G. (2004). A voxel-based approach to gray matter asymmetries. Neurolmage, 22(2), 656-664. https://doi.org/10.1016/j.neuroimage.2004.01.032

Maller, J. J., Anderson, R. J., Thomson, R. H., Daskalakis, Z. J., Rosenfeld, J. V., \& Fitzgerald, P. B. (2017). Occipital bending in schizophrenia. Australian and New Zealand Journal of Psychiatry, 51(1), 32-41. https:// doi.org/10.1177/0004867416642023

Maller, J. J., Thomson, R. H., Rosenfeld, J. V., Anderson, R., Daskalakis, Z. J., \& Fitzgerald, P. B. (2014). Occipital bending in depression. Brain, 137(Pt 6), 1830-1837. https://doi.org/10.1093/brain/awu072

Paus, T., Tomaiuolo, F., Otaky, N., MacDonald, D., Petrides, M., Atlas, J., \& Evans, A. C. (1996). Human cingulate and paracingulate sulci: Pattern, variability, asymmetry, and probabilistic map. Cerebral Cortex, 6(2), 207-214. https://doi.org/10.1093/cercor/6.2.207

Rubens, A. B., Mahowald, M. W., \& Hutton, J. T. (1976). Asymmetry of the lateral (sylvian) fissures in man. Neurology, 26(7), 620-624. https:// doi.org/10.1212/WNL.26.7.620

Toga, A. W., \& Thompson, P. M. (2003). Mapping brain asymmetry. Nature Reviews Neuroscience, 4, 37-48. https://doi.org/10.1038/nrn1009 
Warren, J. (1980). Handedness and laterality in humans and other animals. Physiological Phychology, 8, 351-359. https://doi.org/10.3758/ BF03337470

Watkins, K. E., Paus, T., Lerch, J. P., Zijdenbos, A., Collins, D. L., Neelin, P., \& Evans, A. C. (2001). Structural asymmetries in the human brain: A voxel-based statistical analysis of $142 \mathrm{MRI}$ scans. Cerebral Cortex, 11(9), 868-877. https://doi.org/10.1093/cercor/11.9.868

Xiang, L., Crow, T. J., \& Roberts, N. (2019). Cerebral torque is human specific and unrelated to brain size. Brain Structure and Function (accepted). https://doi.org/10.1007/s00429-018-01818-0

Xiang, L., Crow, T. J., Hopkins, W. D., Gong, Q., \& Roberts, N. (2018). Human torque is not present in chimpanzee brain. Neurolmage, 165 , 285-293. https://doi.org/10.1016/j.neuroimage.2017.10.017
Zilles, K., Dabringhaus, A., Geyer, S., Amunts, K., Qu, M., Schleicher, A., \& Steinmetz, H. (1996). Structural asymmetries in the human forebrain and the forebrain of non-human primates and rats. Neuroscience and Biobehavioral Reviews, 20(4), 593-605. https://doi. org/10.1016/0149-7634(95)00072-0

How to cite this article: Xiang L, Crow TJ, Roberts N.

Automatic analysis of cross-sectional cerebral asymmetry on $3 \mathrm{D}$ in vivo MRI scans of human and chimpanzee. J Neuro Res.

2019;00:1-10. https://doi.org/10.1002/jnr.24391 